\title{
Alta prevalencia de discapacidad en adultos mayores de 15 departamentos de Honduras, año 2015
}

\author{
High prevalence of disability in older adults in 15 departments of Honduras, year 2015
}

\author{
Melissa A. Mejía, (10) 1 Paola M. Rivera, ${ }^{2}$ Melvin Urbina, ${ }^{3}$ Leonardo Sierra, ${ }^{4}$ Sonia Flores, $\left({ }^{5}\right.$ Claudia Martínez,,${ }^{4}$ Saira Rápalo, ${ }^{5}$ \\ Lucía Zúñiga, ${ }^{6}$ José E. Sierra, ${ }^{4}{ }^{4}$ Jackeline Alger,(1) ${ }^{7}$ Mauricio Gonzales, ${ }^{8}$ Edna Maradiaga. ${ }^{9}$
}

${ }^{1}$ Médico Fisiatra, Postgrado de Medicina de Rehabilitación (PMR), Facultad de Ciencias Médicas (FCM), Universidad Nacional Autónoma de Honduras (UNAH); Tegucigalpa; ${ }^{2}$ Médico Fisiatra, Centro de Rehabilitación Integral Teletón; San Pedro Sula; ${ }^{3}$ Médico Fisiatra, Centro de Rehabilitación Integral del Litoral Atlántico (CRILA); ${ }^{4}$ Médico Fisiatra, PMR, FCM UNAH; Tegucigalpa; ${ }^{5}$ Médico Fisiatra, Unidad de Medicina Física y Rehabilitación, Instituto Hondureño de Seguridad Social (IHSS); Tegucigalpa; ${ }^{6}$ Médico Fisiatra, Centro de Rehabilitación Integral Teletón; San Pedro Sula; ${ }^{7} M D$, PhD, Unidad de Investigación Cientíica (UIC) FCM UNAH; Hospital Escuela Universitario; Tegucigalpa; ${ }^{8}$ Licenciado en Informática; UIC FCM UNAH; Tegucigalpa; ${ }^{9}$ MD, Maestría en Salud Pública, UIC FCM UNAH; Tegucigalpa.

RESUMEN. Antecedentes: La prevalencia mundial de discapacidad en adultos mayores oscila entre $29.5 \%$ y $43.4 \%$ en países de ingreso alto/bajo (OMS 2011). Objetivo: Determinar la prevalencia de discapacidad y factores asociados en adultos mayores, Honduras, 2014-2015. Métodos: Estudio descriptivo transversal con análisis de asociación. Estudiantes de último año, Carrera de Medicina, Universidad Nacional Autónoma de Honduras, realizaron búsqueda activa de 50 participantes $\geq 60$ años de edad. Se aplicaron dos instrumentos, uno para búsqueda activa y otro para caracterizar al participante con/sin discapacidad permanente $\geq 1$ año de evolución. Se evaluó limitación de actividad y restricción de participación $\leq 30$ días utilizando la Clasificación Internacional del Funcionamiento, Discapacidad y Salud (CIF) y cuestionario WHODAS 2.0 (OMS). Se realizó análisis univariado (frecuencias, porcentajes, prevalencias, IC95\%) y análisis bivariado (diferencia de proporciones); valor $p<0.05$ se consideró significativo). Resultados: De un total de 5,126 participantes $\geq 60$ años de edad, 3,017 (58.9\%) mujeres, edad promedio 73.6 años (rango 60-106), la prevalencia de discapacidad fue $51.2 \%(2,627 / 5,126)$; discapacidad grado leve se presentó en 1,893 (72.1\%). Las estructuras relacionadas con movimiento $(75.5 \%)$ y función esquelética (72.6\%) fueron las más afectadas. La limitación de moverse $(93.4 \%$ ) y la restricción en invertir dinero propio y familiar en su estado de salud (86.8\%) fueron las más frecuentes. Los factores sexo femenino, edad $>70$ años, escolaridad $<6$ años, enfermedades crónicas, trauma, accidente o violencia podrían estar asociados a discapacidad; todos $p<0.01$. Discusión: La discapacidad en adultos mayores se asoció a algunas condiciones prevenibles. Es apremiante implementar programas de envejecimiento saludable en Honduras.

Palabras clave: Adulto Mayor; Clasificación Internacional del Funcionamiento, de la Discapacidad y de la Salud; Enfermedad Crónica; Evaluación de la Discapacidad; Personas con Discapacidad.

Recibido: 27-05-2020 Aceptado: 19-04-2021 Primera vez publicado en línea: 24-05-2021 Dirigir correspondencia a: Dra. Melissa Mejía

Correo electrónico: meli mcmejia@yahoo.com

Declaración de relaciones y actividades financieras y no financieras y conflictos de interés: ninguno.

DOI: https://doi.org/10.5377/rmh.v89i1.11576

(C) 2021 Autor(es): (c) (i)

\section{INTRODUCCIÓN}

La discapacidad, un problema de salud en aumento, se define como las deficiencias en la estructura o función, limitaciones de la actividad y restricciones a la participación en cualquier grado de severidad. ${ }^{1}$ La Organización Mundial de la Salud (OMS) en su informe Mundial de Discapacidad (2011) estimó que el $15 \%$ de la población mundial presenta algún grado de discapacidad, siendo el riesgo de discapacidad más alto a mayor edad. ${ }^{2}$ En este informe se describe una prevalencia mundial de discapacidad en adultos mayores de $38.1 \%$, con importantes diferencias entre países de ingreso alto (29.5\%) comparados con países de ingreso bajo $(43.4 \%) .{ }^{2}$ La estructura poblacional mundial está en un proceso de transición de una población joven a un grupo de mayor edad y este cambio demográfico ocurre a mayor velocidad en los países de ingreso bajo que en los de ingreso alto. ${ }^{3}$

Envejecer es la evolución de la vida, no una enfermedad, y debido al fenómeno de envejecimiento poblacional, uno de los principales retos a enfrentar en el futuro próximo será la alta prevalencia de discapacidad. ${ }^{4,5} \mathrm{La}$ OMS señala que algunas enfermedades crónicas como las cardiovasculares, diabetes mellitus y cáncer, representan $66.5 \%$ del total de años vividos con discapacidad en países de ingresos medios y bajos. ${ }^{2}$ En México, en el 2012 se estimó que el $47.8 \%$ de los adultos mayores tenía algún grado de discapacidad. Cifras similares se reportaron en Chile $45.0 \%$ y en Nicaragua $30.0 \%$. ${ }^{6,7}$ Entre los factores asociados a discapacidad en este grupo poblacional, prevalecen las caídas, mayor edad, sexo femenino, bajo nivel educacional, evento cerebrovascular, artrosis, deterioro cognoscitivo, cardiopatías y depresión, entre otros. ${ }^{3,8,9}$ En Chile se concluyó que las mujeres presentan mayor frecuencia de discapacidad severa comparada con hombres de su misma edad. ${ }^{7}$

En Honduras, esta misma tendencia de mayor frecuencia de discapacidad a mayor edad se reflejó en la Encuesta Permanente de Hogares de Propósitos Múltiples realizada por el Instituto Nacional de Estadística (INE 2002), informando 
que $31.0 \%$ de la población con discapacidad correspondió a adultos mayores de 65 años y la prevalencia de discapacidad en adultos mayores fue $19.0 \% .{ }^{10}$ Considerando que estas cifras tienen casi veinte años y debido a la carencia de información más reciente, se realizó este estudio con el objetivo de determinar la prevalencia de discapacidad y los factores asociados a la discapacidad en adultos mayores con el fin de contribuir al desarrollo de políticas públicas necesarias para la implementación de programas de envejecimiento saludable y prevención de discapacidad, que mejoren la calidad de vida y participación social del adulto mayor en Honduras.

\section{PARTICIPANTES Y MÉTODOS}

Se realizó un estudio descriptivo transversal con análisis de asociación en personas con edad igual o mayor a 60 años, residentes en las áreas geográficas de influencia (AGI) de los estudiantes del último año de la Carrera de Medicina de la Facultad de Ciencias Médicas (FCM), Universidad Nacional Autónoma de Honduras (UNAH), del periodo 2014 - 2015. Los estudiantes cursaban el Seminario de Investigación impartido por la Unidad de Investigación Científica (UIC), como requisito de graduación y fueron distribuidos en todo el país. Los datos fueron recolectados en los meses de octubre del 2014 a marzo del 2015.

La muestra de personas adulto mayor se calculó estimando una población total de 8 millones de hondureños (INE), ${ }^{11}$ aproximadamente $7.5 \%$ con edad igual o mayor a 60 años $(600,000)$, distribuyéndose aproximadamente el $50.0 \%$ (4 millones de personas de población general y 300,000 de adultos mayores) viviendo en zonas urbanas y rurales, respectivamente. Tomando una prevalencia máxima de discapacidad de $31.0 \%$ y mínima de $25.0 \%$, con un $80.0 \%$ de poder estadístico y $95.0 \%$ de nivel de confianza, se estimó una muestra de 1,000 adultos mayores, la que se incrementó a 2,000 para contar con poder estadístico de estratificación y reducir el sesgo de selección. Cada estudiante entrevistó a 50 adultos mayores.

Todos los estudiantes asignados a su respectiva unidad de salud, en comunidades urbanas y rurales, realizaron muestreo no probabilístico de manera coordinada a través de los siguientes pasos: a) Elaboración de un listado enumerado de todas las localidades del AGI con una población total que aseguró que la población de adultos mayores fuera 50 o más y condiciones de seguridad física aceptables para el entrevistador; b) Selección aleatoria de una localidad por cada estudiante asignado a la unidad de salud; c) Búsqueda activa de casos, mediante visita casa a casa (barrido), hasta cumplir con el tamaño muestral de 50 adultos mayores. Se definió como caso de discapacidad toda persona igual o mayor a 60 años de edad con un año o más de presentar alteración en la estructura y función, limitaciones en la actividad y restricciones en la participación de cualquier grado de severidad medido a través del cuestionario desarrollado por la OMS denominado WHODAS 2.0 por sus siglas en inglés (WHO Disability Assessment Schedule 2.0). ${ }^{12}$ Se incluyeron los participantes de acuerdo a la definición de caso y que aceptaron participar voluntariamente. A todos los participantes a quienes se les detectó discapacidad, se les explicó la importancia de la rehabilitación, se le refirió al establecimiento de salud correspondiente y al centro de rehabilitación más cercano y se les proporcionó un trifolio informativo (ver http://www.bvs.hn/Honduras/UICFCM/TRIFOLIO DISCAPACIDAD.pdf)

Los estudiantes participaron en seis talleres sobre temas de metodología de investigación, discapacidad y rehabilitación, y ética de la investigación, con el fin de capacitarlos en la identificación de personas con discapacidad y el adecuado registro de la información, en los instrumentos que aplicaron. La información fue registrada en dos instrumentos: uno para registro de las viviendas y datos generales de la población (Instrumento 1) y otro para identificar a las personas con discapacidad, el cual se basó en el Cuestionario WHODAS 2.0 y se aplicó a todo adulto mayor entrevistado (Instrumento 2). ${ }^{12,13}$

El cuestionario WHODAS 2.0 está basado en una conceptualización de la discapacidad que puede ser aplicada de manera universal a las condiciones de salud. Para su análisis se utilizó la Clasificación Internacional del Funcionamiento de la Discapacidad y de la Salud (CIF), ${ }^{1}$ que permitió conocer los diferentes niveles de deficiencia y valorar el grado de discapacidad en los últimos 30 días. El cuestionario se compone de seis dimensiones: comprensión y comunicación, capacidad para moverse, cuidado personal, relacionarse con otras personas, actividades de la vida diaria y participación. Para cada dimensión hay una serie de preguntas y en cada pregunta, el puntaje mínimo es cero y el máximo 4, luego se realiza sumatoria de las preguntas en cada dimensión para obtener la severidad de afección en cada dimensión, y finalmente se suman los puntos obtenidos en todas las dimensiones para calcular la severidad de la discapacidad en el sujeto y para ello se utiliza la siguiente escala: $0=$ No hay problema (0-4\%), $1=$ Problema leve $(5-24 \%)$, 2= Problema moderado (25-49\%), 3= Problema grave (50-95\%), 4= Problema completo (96-100\%). Se estimó el porcentaje final y según el resultado se clasificó según CIF en discapacidad leve, moderada, grave o completa. La discapacidad identificada se clasificó según su causa en congénita 0 adquirida. ${ }^{14}$

Se realizaron dos pruebas piloto para validar la comprensión del contenido de los instrumentos. En la primera, los dos instrumentos fueron validados por cada estudiante de último año de la Carrera de Medicina mediante entrevista a cinco adultos mayores en una localidad diferente a la seleccionada. En la segunda, cada uno de los médicos residentes del Postgrado de Medicina de Rehabilitación (PMR) del equipo de investigación validaron el Instrumento 2, con cinco adultos mayores con discapacidad captados en establecimientos de salud en Tegucigalpa. Las inconsistencias y recomendaciones para mejorar la comprensión fueron informadas al grupo coordinador del estudio y se realizaron los cambios pertinentes. Los participantes adultos mayores detectados con discapacidad en esta fase del estudio fueron remitidos a la unidad de rehabilitación más cercana.

Se realizó control de calidad de la información registrada cuando los miembros del grupo coordinador, médicos residentes del PMR y médicos fisiatras colaboradores, supervisaron la recolección de la información por parte de los estudiantes 
en una muestra por conveniencia de 28 ciudades visitadas durante la recolección de datos. Adicionalmente, los instrumentos fueron recibidos en dos centros de recolección ubicados estratégicamente en las dos ciudades principales del país, San Pedro Sula y Tegucigalpa. Los instrumentos recibidos fueron revisados y los estudiantes con instrumentos incompletos y/o información inconsistente fueron contactados y procedieron a completar la información y sometieron nuevamente sus resultados. Finalmente, los médicos residentes de tercer año realizaron depuración de los instrumentos revisados. La depuración consistió en corroborar el número de identidad de las personas entrevistadas con el censo nacional y seleccionar para análisis los instrumentos de los estudiantes que presentaron información completa o que presentaron $\leq 5$ instrumentos incompletos y/o con información inconsistente. Se creó una base de los datos y estos se procesaron con el paquete estadístico Epilnfo 7.0 (versión Windows) en la UIC FCM UNAH. Se realizó análisis univariado para las características de la población, las limitaciones y el grado de discapacidad mostradas como frecuencias y porcentajes. La prevalencia se estimó utilizando la fórmula $\mathrm{P}=$ número de personas con discapacidad / número de habitantes igual o mayor de 60 años de edad, multiplicado por 100, con su respectivo intervalo de confianza del 95\% (IC95\%). Se realizó análisis bivariado para las deficiencias de personas con discapacidad por sexo y se calculó diferencia de proporciones $\left(\mathrm{X}^{2}\right)$ para las característica generales y antecedentes en adultos mayores con o sin discapacidad.

El protocolo contó con dictamen del Comité de Ética en Investigación Biomédica (CEIB), FCM UNAH. A cada participante se le explicó el propósito del estudio y se obtuvo consentimiento informado escrito, garantizando la confidencialidad de la información personal de los participantes. Si el participante no era capaz de comprender la información brindada y responder por sí mismo, un familiar o encargado mayor de 18 años tomó la decisión de su participación. Tanto los médicos residentes y los estudiantes fueron capacitados mediante el curso en línea de Buenas Prácticas Clínicas (Programa CITI, Universidad de Miami, www.citiprogram.org).

\section{RESULTADOS}

Un total de 541 estudiantes se registraron en el Seminario de Investigación 2014 - 2015 y completaron el proceso de recolección de información. Después del proceso de revisión y depuración de los instrumentos, se incluyeron 107 (19.8\%) estudiantes que presentaron $\leq 5$ instrumentos incompletos y/o con información inconsistente. Estos 107 estudiantes visitaron 8,202 casas identificando un total de 29,968 personas; de éstos, $5,126(17.1 \%)$ eran personas adulto mayor distribuidos en 15 de los 18 departamentos de Honduras, sin incluir los departamentos de Comayagua, Gracias a Dios e Islas de la Bahía. Del total de 5,126 personas adulto mayor, se identificaron 2,627 con discapacidad para una prevalencia de discapacidad en personas adulto mayor de $51.2 \%$ (IC95\% 49.9-52.6) en la muestra estudiada (Ver diagrama de flujo en Figura 1).
Del total de 2,627 adultos mayores con discapacidad, el promedio de edad fue 73.6 años (rango 60-106), predominó el sexo femenino $62.6 \%(1,645), 77.2 \%(2,029)$ procedía de área urbana, estado civil soltero $53.5 \%(1,406)$ y nivel de escolaridad $\leq 6$ años $90.6 \%(2,380)$ (Cuadro 1). La discapacidad fue adquirida en $91.6 \%(2,408)$ de los participantes. Entre las causas adquiridas, $79.1 \%(1,904)$ fue por enfermedad común y $7.8 \%$ (188) por trauma. El 94.5\% $(2,482)$ informó vivir acompañado por el conyugue, hijo u otro. El 9.8\% (258) informó haber participado en algún momento en un Programa de Rehabilitación (Cuadro 1).

En relación con la limitación en la actividad y grado de severidad, según orden de frecuencia principalmente se identificó limitación en la capacidad para moverse en $93.4 \%(2,454)$, en actividades de la vida diaria $82.7 \%(2,173)$ y comprensión y comunicación $75.0 \%$ (1,969) (Cuadro 2). En la capacidad para moverse, la actividad más frecuentemente limitada fue caminar largas distancias como 1 kilómetro $(2,208)$, con aproximadamente un tercio de los afectados en grado leve. Las actividades de la vida diaria fueron mayormente limitadas en finalizar con los quehaceres de la casa tan rápido como fuera necesario $(2,066)$, con aproximadamente la mitad afectados en grado leve. De los que presentaron alguna limitación en la comprensión y comuni-

Figura 1. Diagrama de flujo del proceso de búsqueda activa de casos de personas adulto mayor con discapacidad, Honduras, 2014-2015.

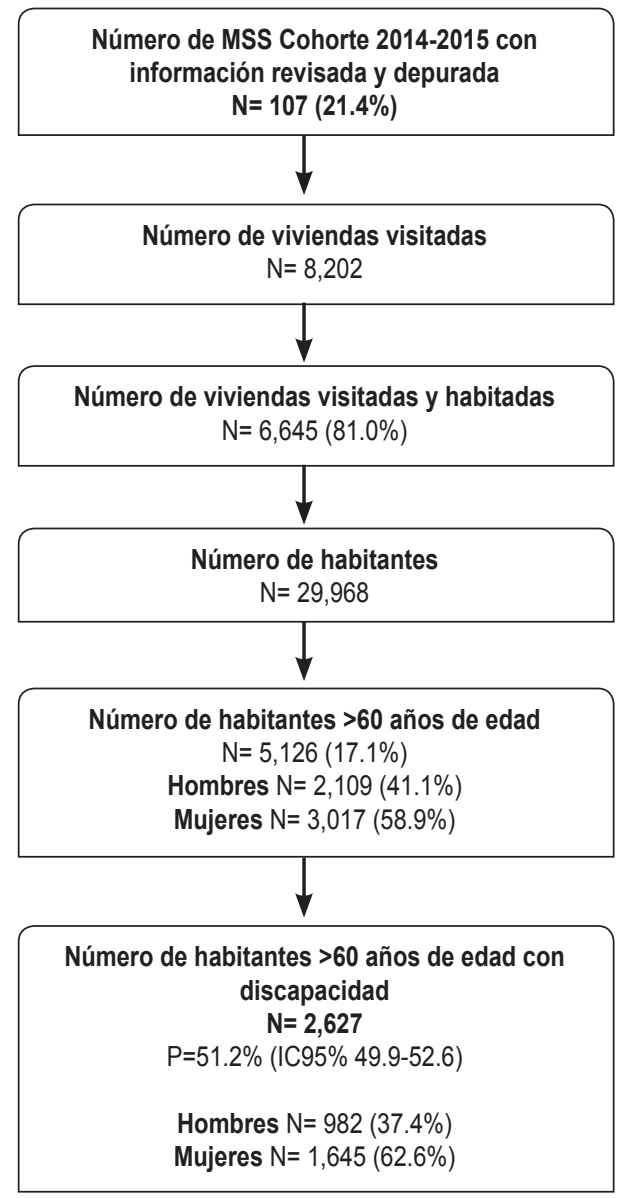


Cuadro 1. Distribución de participantes personas adulto mayor con discapacidad de acuerdo a características sociodemográficas y antecedentes, Honduras, 2014-2015, $n=2,627$.

\begin{tabular}{|c|c|}
\hline Características & $\mathrm{N}(\%)$ \\
\hline $\begin{array}{l}\text { Edad (años) } \\
60-69 \\
70-79 \\
\geq 80 \\
\text { Promedio } \\
\text { Rango }\end{array}$ & $\begin{array}{c}1,008(38.4) \\
903(34.4) \\
716(27.3) \\
73.6 \\
60-106\end{array}$ \\
\hline $\begin{array}{l}\text { Sexo } \\
\text { Femenino } \\
\text { Masculino }\end{array}$ & $\begin{array}{c}1,645(62.6) \\
982(37.4)\end{array}$ \\
\hline $\begin{array}{l}\text { Estado civil } \\
\text { Soltero } \\
\text { Casado } \\
\text { Unión libre }\end{array}$ & $\begin{array}{l}1,406(53.5) \\
911(34.7) \\
310(11.8)\end{array}$ \\
\hline $\begin{array}{l}\text { Procedencia } \\
\text { Urbano } \\
\text { Rural } \\
\text { No consignado }\end{array}$ & $\begin{array}{c}2029(77.2) \\
569(21.7) \\
29(1.1)\end{array}$ \\
\hline $\begin{array}{l}\text { Vive acompañado } \\
\text { No } \\
\text { Si } \\
\text { Pareja } \\
\text { Hijo/hija } \\
\text { Otro }\end{array}$ & $\begin{array}{c}145(5.5) \\
2,482(94.5) \\
1,152(46.4) \\
1,731(69.7) \\
1,091(44.0)\end{array}$ \\
\hline $\begin{array}{l}\text { Causa } \\
\text { Congénita } \\
\text { Congénita + adquirida } \\
\text { Adquirida } \\
\text { No consignado }\end{array}$ & $\begin{array}{c}3(0.1) \\
7(0.3) \\
2,408(91.6) \\
209(8.0)\end{array}$ \\
\hline $\begin{array}{l}\text { Tipo de discapacidad adquirida } \\
\text { Trauma } \\
\text { Caída } \\
\text { Violencia } \\
\text { Accidente } \\
\text { Enfermedad común } \\
\text { Adquirida no consignada }\end{array}$ & $\begin{array}{c}188(7.8) \\
134(5.6) \\
10(0.4) \\
44(1.8) \\
1,904(79.1) \\
323(13.4)\end{array}$ \\
\hline $\begin{array}{l}\text { Nivel de Instrucción (años) } \\
\leq 6 \\
>6\end{array}$ & $\begin{array}{c}2,380(90.6) \\
247(9.4)\end{array}$ \\
\hline $\begin{array}{l}\text { Participación en Programa de Rehabilitación } \\
\mathrm{Si} \\
\text { No }\end{array}$ & $\begin{array}{c}258(9.8) \\
2,369(90.2)\end{array}$ \\
\hline
\end{tabular}

cación, la actividad más frecuentemente afectada fue recordar cosas importantes $(1,446)$, más de la mitad de los afectados en grado leve. En el cuidado personal, la actividad más limitada fue estar solo unos días $(1,078)$, aproximadamente un tercio de los afectados en grado leve. En el aspecto de relacionarse con otras personas, la actividad más limitada fue relacionarse con personas que no conoce (419), más de la mitad afectados en grado leve. Del total de adultos mayores con discapacidad, todos $(2,627)$ presentaron restricción en la participación en la sociedad. El aspecto más restringido fue haber tenido que invertir dinero de sus ingresos y de su familia en su estado de salud $(2,279)$, cerca de la mitad en grado leve. Seguidamente, haber invertido tiempo para buscar ayuda para su estado de salud se presentó con mayor frecuencia $(2,245)$, cerca de la mitad en grado leve (Cuadro 2).
El grado de severidad de la discapacidad fue leve $72.1 \%$ $(1,893)$, moderado $22.1 \%$ (581), severo $5.7 \%$ (151) y completo $0.1 \%(2)$. El grado de severidad leve presentó una prevalencia de $38.6 \%$ en las mujeres y $24.1 \%$ en los hombres; la severidad moderada-completa presentó una prevalencia de $15.9 \%$ en las mujeres y $8.4 \%$ en los hombres. La participación en un programa de rehabilitación presentó una prevalencia de $5.7 \%$ en las mujeres y $4.1 \%$ en los hombres.

En el Cuadro 3 se presenta las prevalencias de las deficiencias en adultos mayores con discapacidad según sexo. Las funciones con prevalencias más afectadas en ambos sexos fueron esqueléticas $40.9 \%$ (IC95\% 39.2-42.7) y 31.9\% (IC95\% 30.0-34.0) para sexo femenino y masculino, respectivamente, y cardiovascular $36.4 \%$ (IC95\% 34.7-38.1) y $23.7 \%$ (IC95\% 21.925.5) para sexo femenino y masculino, respectivamente. Las estructuras mas afectadas en ambos sexos fueron las relacionadas al movimiento $42.0 \%$ (IC95\% 40.3-43.8) y 33.9\% (IC95\% 31.936.0) para sexo femenino y masculino, respectivamente, y sistema cardiovascular $36.0 \%$ (IC95\%34.3-37.7) y $23.5 \%$ (IC95\% 21.7-25.3) para sexo femenino y masculino, respectivamente.

En relación con características generales, el tener menos de 6 años de instrucción escolar estuvo presente en el $90.6 \%$ de los que tenían discapacidad $(p<0.01)$, tener alguna enfermedad crónica $84.6 \%(p<0.01)$, entre ellas la hipertensión arterial $59.3 \%(p<0.01)$, artropatía $42.3 \%(p<0.01)$ y diabetes mellitus $20.3 \%(p<0.01)$, y el sexo femenino $62.6 \%(p<0.01)$, ver Cuadro 4. Todas las características analizadas entre personas adulto mayor con y sin discapacidad mostraron diferencias de proporción con significancia estadística, excepto vivir solo y antecedentes de cáncer y de accidentes.

\section{DISCUSIÓN}

En este estudio a través de la búsqueda activa de una muestra por conveniencia en 15 de los 18 departamentos de Honduras, se ha identificado una prevalencia de discapacidad en personas adulto mayor de $51.2 \%$, la cual supera 2.7 veces la informada por el INE en el año $2002(19.0 \%))^{10}$ Esta diferencia podría explicarse debido a las diferencias en el personal que realizó la recolección de la información, el proceso de revisión de los datos y el instrumento que se utilizó para la identificación de la discapacidad (WHODAS 2.0). También, podría deberse al aumento en la última década de enfermedades crónicas en el país, lo que podría contribuir a un aumento en la discapacidad en el adulto mayor. ${ }^{11}$ Por otra parte, esta prevalencia identificada en nuestro estudio es inferior a la prevalencia informada en Perú $(59.8 \%)$ y superior a México (47.8\%), Chile (45.0\%), Nicaragua $(30.0 \%)$ y Marruecos $(33.2 \%))^{7,15,16}$ En relación al grado de discapacidad identificado el cual fue leve en $72.1 \%$, moderado en $22.1 \%$, severo en $5.7 \%$ y completo en $0.1 \%$, hay similitud a lo informado por OMS (2011) para población general: grado leve en $81.8 \%$, moderado en $15.3 \%$ y grave en $2.9 \%$, aunque no se describe el grado de discapacidad completo. ${ }^{2}$ El grado de discapacidad identificado en nuestro estudio concuerda con la tendencia mundial. ${ }^{2}$ Aunque la prevalencia sea alta, la mayoría presenta una discapacidad leve. 
Cuadro 2. Distribución de participantes con problemas de limitación en la actividad y restricción en la participación en la sociedad según el grado de severidad de la dimensión afectada en personas adulto mayor con discapacidad, Honduras, 2014-2015, n=2,627.

\begin{tabular}{|c|c|c|c|c|c|c|}
\hline \multirow{2}{*}{$\begin{array}{l}\text { PARTICIPANTES CON PROBLEMAS EN } \\
\text { LAS SIGUIENTES ÁREAS }\end{array}$} & \multicolumn{6}{|c|}{ GRADO DE SEVERIDAD DE LA DIMENSIÓN AFECTADA } \\
\hline & $\begin{array}{c}\text { Ninguna } \\
\mathrm{N}(\%)\end{array}$ & $\begin{array}{l}\text { Algún grado } \\
\mathrm{N}(\%)\end{array}$ & $\begin{array}{c}\text { Leve } \\
\mathrm{N}\end{array}$ & $\begin{array}{c}\text { Moderada } \\
\mathrm{N}\end{array}$ & $\begin{array}{c}\text { Severa } \\
\mathrm{N}\end{array}$ & $\underset{\mathrm{N}}{\text { Completo }}$ \\
\hline Comprensión y comunicación & $658(25.0)$ & $1,969(75.0)$ & -- & -- & -- & -- \\
\hline 1. Concentrarse y hacer algo $10 \mathrm{~min}$ & -- & 1,084 & 686 & 310 & 67 & 21 \\
\hline 2. Recordar cosas importantes & -- & 1,446 & 873 & 453 & 91 & 29 \\
\hline 3. Aprender una nueva tarea como llegar a un lugar donde no ha estado & -- & 1,344 & 655 & 460 & 163 & 66 \\
\hline Capacidad para moverse & $173(6.6)$ & $2,454(93.4)$ & -- & -- & -- & -- \\
\hline 1. Estar de pie durante $30 \mathrm{~min}$ & -- & 1,942 & 924 & 723 & 224 & 71 \\
\hline 2. Ponerse de pie cuando estaba sentado & -- & 1,675 & 945 & 533 & 150 & 47 \\
\hline 3. Caminar distancias como $1 \mathrm{~km}$ & -- & 2,208 & 788 & 841 & 391 & 188 \\
\hline Cuidado personal & $1,312(49.9)$ & $1,315(50.1)$ & -- & -- & -- & -- \\
\hline 1. Bañarse & -- & 576 & 306 & 155 & 79 & 36 \\
\hline 2. Vestirse & -- & 520 & 288 & 133 & 62 & 37 \\
\hline 3. Estar solo unos días & -- & 1,078 & 424 & 339 & 199 & 116 \\
\hline Relacionarse con otras personas & $2,012(76.6)$ & $615(23.4)$ & -- & -- & -- & -- \\
\hline 1. Relacionarse con personas que no conoce & -- & 419 & 241 & 120 & 43 & 15 \\
\hline 2. Mantener una amistad & -- & 217 & 106 & 69 & 31 & 11 \\
\hline 3. Hacer nuevos amigos & -- & 360 & 202 & 90 & 50 & 18 \\
\hline Actividades de la vida diaria & $454(17.3)$ & $2,173(82.7)$ & - & -- & -- & -- \\
\hline 1. Realizar bien los quehaceres de la casa más importantes & -- & 1,633 & 942 & 421 & 146 & 124 \\
\hline 2. Acabar todo el trabajo de la casa que tenía que hacer & -- & 1,802 & 951 & 545 & 178 & 128 \\
\hline 3. Acabar con los quehaceres de la casa tan rápido como sea necesario & -- & 2,066 & 1,024 & 666 & 237 & 139 \\
\hline \multicolumn{7}{|l|}{ RESTRICCIÓN PARA } \\
\hline Participación en la sociedad & -- & & -- & -- & -- & -- \\
\hline 1. Participa en el mismo nivel que el resto en las actividades de la comunidad & -- & 1,855 & 1,010 & 523 & 224 & 98 \\
\hline 2. Ha invertido tiempo para buscar ayuda para su estado de salud & -- & 2,245 & 1,114 & 824 & 240 & 67 \\
\hline $\begin{array}{l}\text { 3. Ha tenido que invertir dinero de sus ingresos y de su familia en su estado } \\
\text { de salud }\end{array}$ & - & 2,279 & 1,077 & 874 & 256 & 72 \\
\hline
\end{tabular}

Cuadro 3. Prevalencia de las deficiencias (funciones/estructuras) en adultos mayores con discapacidad por sexo, Honduras 2014-2015, n=2,627.

\begin{tabular}{|c|c|c|c|c|c|c|}
\hline \multirow{2}{*}{$\begin{array}{l}\text { Deficiencias } \\
\text { (Función/Estructura) }\end{array}$} & \multicolumn{3}{|c|}{$\begin{array}{l}\text { Adultos mayores con discapacidad por sexo } \\
\text { Número }\end{array}$} & \multicolumn{3}{|c|}{$\begin{array}{l}\text { Prevalencia de discapacidad por sexo } \\
\qquad \%(\text { IC95\%) }\end{array}$} \\
\hline & $\begin{array}{l}\text { Hombre } \\
\mathrm{n}=982\end{array}$ & $\begin{array}{c}\text { Mujer } \\
\mathrm{n}=1,645\end{array}$ & Total & $\begin{array}{l}\text { Hombre } \\
n=2,109\end{array}$ & $\begin{array}{c}\text { Mujer } \\
\mathrm{n}=3,017\end{array}$ & $\begin{array}{c}\text { Total } \\
n=5,126\end{array}$ \\
\hline \multicolumn{7}{|l|}{ Funciones } \\
\hline Mentales & 473 & 830 & 1,303 & $22.4(20.7-24.2)$ & $27.1(25.9-29.1)$ & $25.4(24.2-26.6)$ \\
\hline Sensoriales y dolor & 541 & 919 & 1,460 & $25.6(23.8-27.5)$ & $30.5(28.8-32.1)$ & $28.5(27.2-29.7)$ \\
\hline Cardiovascular & 499 & 1,098 & 1,597 & $23.7(21.9-25.5)$ & $36.4(34.7-38.1)$ & $31.1(29.9-32.4)$ \\
\hline Digestiva & 108 & 223 & 331 & $5.1(4.2-6.1)$ & $7.4(6.5-8.4)$ & $6.4(5.8-7.1)$ \\
\hline Respiratoria & 142 & 182 & 324 & $6.7(5.7-7.9)$ & $6.0(5.2-6.9)$ & $6.3(5.7-7.0)$ \\
\hline Metabólica & 146 & 341 & 487 & $6.9(5.9-8.1)$ & $11.3(10.2-12.5)$ & $9.5(8.2-10.3)$ \\
\hline Endócrina & 154 & 368 & 522 & $7.3(6.2-8.5)$ & $12.2(11.1-13.4)$ & $10.2(9.4-11.3)$ \\
\hline Neuromuscular & 267 & 403 & 670 & $12.7(11.3-14.1)$ & $13.3(12.2-14.6)$ & $13.1(12.2-14.0)$ \\
\hline Esquelética & 674 & 1,234 & 1,908 & $31.9(30.0-34.0)$ & $40.9(39.2-42.7)$ & $37.2(35.9-38.5)$ \\
\hline \multicolumn{7}{|l|}{ Estructuras } \\
\hline Sistema Nervioso & 500 & 884 & 1,384 & $23.7(21.9-25.6)$ & $29.3(27.7-30.9)$ & $27.0(25.8-28.2)$ \\
\hline Ojo y relacionados & 366 & 631 & 997 & $17.3(15.8-19.0)$ & $20.9(19.5-22.4)$ & $19.4(18.4-20.5)$ \\
\hline Oído y relacionados & 189 & 214 & 403 & $9.0(7.9-10.2)$ & $7.1(6.2-8.0)$ & $7.9(7.1-8.6)$ \\
\hline Cardiovascular & 495 & 1,086 & 1,581 & $23.5(21.7-25.3)$ & $36.0(34.3-37.7)$ & $30.8(29.6-32.1)$ \\
\hline Respiratorio & 136 & 175 & 311 & $6.4(5.4-7.5)$ & $5.8(5.0-6.7)$ & $6.1(5.4-6.7)$ \\
\hline Digestivo & 103 & 224 & 327 & $4.9(4.0-5.9)$ & $7.4(6.5-8.4)$ & $6.4(5.7-7.1)$ \\
\hline Metabólica & 145 & 314 & 459 & $6.9(5.8-8.0)$ & $10.4(9.3-11.5)$ & $8.9(8.2-9.7)$ \\
\hline Endócrina & 161 & 332 & 493 & $7.6(6.5-8.8)$ & $11.0(9.9-12.2)$ & $9.6(8.8-10.4)$ \\
\hline Genitourinario y reproductor & 205 & 153 & 537 & $9.7(8.5-11.0)$ & $5.1(4.3-5.9)$ & $10.5(9.6-11.3)$ \\
\hline Relacionadas al movimiento & 716 & 1,268 & 1,984 & $33.9(31.9-36.0)$ & $42.0(40.3-43.8)$ & $38.7(37.4-40.0)$ \\
\hline
\end{tabular}

No se incluyeron las deficiencias con prevalencias menores a $5 \%$ (Funciones/estructuras de la voz y el habla, inmunológica y de piel y relacionados; y funciones hematológica, genitourinaria y reproductora) 
Cuadro 4. Diferencia de proporciones en adultos mayores con/sin discapacidad según características generales y antecedentes , Honduras, 2014-2015, $n=5,126$.

\begin{tabular}{|c|c|c|c|}
\hline \multirow[b]{2}{*}{ FACTORES } & \multicolumn{2}{|c|}{ DISCAPACIDAD } & \multirow[b]{2}{*}{ Valor $\mathrm{p}^{*}$} \\
\hline & $\begin{array}{c}\text { Presente } \\
\mathrm{N}=2,627 \\
\mathrm{~N}(\%)\end{array}$ & $\begin{array}{c}\text { Ausente } \\
\mathrm{N}=2,499 \\
\mathrm{~N}(\%)\end{array}$ & \\
\hline Sexo Femenino & $1,645(62.6)$ & $1,372(54.9)$ & $<0.01$ \\
\hline Edad $\geq 70$ años & $1,500(57.1)$ & $921(36.9)$ & $<0.01$ \\
\hline Años de instrucción $\leq 6$ & $2,380(90.6)$ & $2,101(84.1)$ & $<0.01$ \\
\hline Vivir solo & $145(5.5)$ & $141(5.6)$ & 0.8 \\
\hline $\begin{array}{l}\text { Antecedentes } \\
\text { Enfermedad crónica }\end{array}$ & $2,222(84.6)$ & $1,425(57.0)$ & $<0.01$ \\
\hline Diabetes mellitus & $533(20.3)$ & $336(13.4)$ & $<0.01$ \\
\hline Hipertensión Arterial & $1,558(59.3)$ & $1,012(40.5)$ & $<0.01$ \\
\hline $\mathrm{ACV}^{*+}$ & $122(4.6)$ & $34(1.4)$ & $<0.01$ \\
\hline Cardiopatía & $279(10.6)$ & $84(3.4)$ & $<0.01$ \\
\hline Artropatía & $1,111(42.3)$ & $453(18.1)$ & $<0.01$ \\
\hline $\mathrm{EPOC}^{+*+}$ & $214(8.1)$ & $72(2.9)$ & $<0.01$ \\
\hline Cáncer & $39(1.5)$ & $24(1.0)$ & 0.08 \\
\hline Trauma & $188(7.2)$ & $85(3.4)$ & $<0.01$ \\
\hline $\begin{array}{l}\text { Caídas en últimos } 12 \\
\text { meses }\end{array}$ & $720(27.4)$ & $383(15.3)$ & $<0.01$ \\
\hline Violencia & $25(1.0)$ & $7(0.3)$ & $<0.01$ \\
\hline Accidente & $95(3.6)$ & $70(2.8)$ & 0.1 \\
\hline
\end{tabular}

"Estadístico $\mathrm{X}^{2}$; ${ }^{* *} \mathrm{ECV}=$ Enfermedad Cerebrovascular; ${ }^{* *} \mathrm{EPOC}=$ Enfermedad Pulmonar Obstructiva Crónica.

Los factores asociados significativamente a discapacidad en adultos mayores identificados en este estudio, en su orden, fueron antecedentes de enfermedad crónica, entre las que ocupa el primer lugar la hipertensión arterial (HTA) seguida por la artropatía, diabetes mellitus, la enfermedad cardiovascular (cardiopatía y enfermedad cerebrovascular) y enfermedad pulmonar obstructiva crónica (EPOC); y factores sociodemográficos como violencia, edad $\geq 70$ años, antecedentes de traumas, caídas en los últimos 12 meses y bajo nivel de escolaridad. En México, las caídas en los últimos 12 meses se identificaron como factor asociado a la discapacidad. ${ }^{8}$ Estos resultados son similares a los reportados en varios estudios de América Latina, donde se encontró asociación de los siguientes factores con discapacidad en el adulto mayor: sexo femenino, mayor edad, menor nivel educacional, artropatías, evento cerebrovascular, cardiopatías, enfermedad pulmonar obstructiva crónica, hipertensión arterial, y diabetes. ${ }^{9,17,18}$ Los factores antes mencionados están directamente asociados a discapacidad en el adulto mayor y la mayoría de ellos son prevenibles.

En cuanto a las características sociodemográficas, el sexo femenino fue el más afectado $62.6 \%$. Este resultado es superior al $54.7 \%$ informado en Perú. ${ }^{16}$ En cuanto a educación, en nuestro estudio $90.6 \%$ de los participantes tenía 6 o menos años de escolaridad, superando a lo reportado en Perú $(71.1 \%)$, Ecuador $(58.8 \%)$, Guatemala $(52.0 \%)$, El Salvador $(44.8 \%)$ y Nicaragua $(45.0 \%){ }^{16}$ Se ha descrito que a menor nivel de educación existe mayor riesgo de discapacidad y es posible que este factor esté contribuyendo a la discapacidad encontrada en nuestro estudio, en el cual el factor escolaridad igual o menor a 6 años se asoció significativamente al grupo con discapacidad. ${ }^{16-18}$

En relación a la limitación en la actividad, las más afectadas fueron la capacidad para moverse (93.6\%), actividades de la vida diaria (82.7\%) y comprensión y comunicación $(75.0 \%)$, superando a lo reportado por Neves y Manrique para varios países de la región..$^{18-20}$ En cuanto a las dificultades para cuidado personal y relacionarse con otras personas, las proporciones identificadas en nuestro estudio son similares a lo encontrado en México (51.0\% y $16.0 \%$, respectivamente). ${ }^{20}$ La gran mayoría de los adultos mayores con discapacidad informó de la necesidad de invertir dinero propio o de su familia para su estado de salud, ${ }^{21}$ reflejando la pobre cobertura en seguridad social para esta población. Según HelpAge International, la cobertura de seguridad social de es apenas $8 \%$ para los adultos mayores hondureños. ${ }^{22}$ Esta situación es muy similar a lo reportado en Chile en 2014, donde $80 \%$ de las personas adulto mayor no son beneficiarios del sistema de salud y por lo tanto, deben utilizar sus propios recursos. ${ }^{23}$ La baja cobertura de seguridad social en Honduras para el adulto mayor limita su acceso a una atención de salud digna y oportuna, llevándolo a invertir su dinero, contribuyendo a una situación de precariedad económica que también conlleva impacto social negativo.

Las prevalencias para las deficiencias tuvieron mayor porcentaje en funciones musculoesqueléticas en primer lugar seguido por cardiovasculares y de igual forma en las estructuras afectadas relacionadas con el movimiento y cardiovasculares respectivamente, encontrando asociación estadísticamente significativa para discapacidad con el sexo femenino. Esto es similar a lo informado por Nusselder y colaboradores quienes encontraron que las enfermedades musculoesqueléticas contribuían a mayor discapacidad en las mujeres mayores de 50 años; sin embargo, en los hombres en las mismas edades esta contribución a la discapacidad era por enfermedades cardiovasculares y respiratorias. ${ }^{24}$

Se encontró concordancia con lo descrito de manera general por Neves y colaboradores para América Latina, sobre la mayor frecuencia de la discapacidad motora. ${ }^{18}$ No obstante, nuestros resultados se pueden comparar con la epidemiología que prevalece por sexo en adultos mayores. Al comparar las mujeres con los hombres, las mujeres presentaron mayor prevalencia de afectación en la función y estructura cardiovascular, contrastando con lo descrito en la literatura donde tienen mayor riesgo los hombres en este tipo de patologías. ${ }^{25}$ Por otra parte, la causa de discapacidad fue adquirida en $91.9 \%$ siendo la enfermedad común la más frecuente $(78.8 \%)$, similar a lo encontrado en Chile (enfermedades crónicas y degenerativas $78.2 \%$ ) y superando lo reportado en 12 países de América Latina incluido Honduras (enfermedades crónicas 63.3\%). 18,19,26,27

A pesar de que en Honduras existe un amplio marco legal a favor de las personas con discapacidad y de su inclusión en la sociedad, ${ }^{28}$ su aplicación real es escasa e insuficiente lo que coincide con un hallazgo inquietante de que solo el $9.8 \%$ de los participantes en situación de discapacidad participó en un 
Programa de Rehabilitación en algún momento. Este resultado es similar a lo encontrado en Chile $(6.5 \%)^{17}$ y es inferior a lo informado por el INE $2002(17.0 \%) .{ }^{10}$ Asimismo está relacionado con la limitada capacidad de respuesta institucional del país en cuanto a infraestructura, aditamentos y personal capacitado en rehabilitación..$^{29}$

Este estudio tiene la limitante de no haber aplicado un muestreo aleatorio. El muestreo a conveniencia incluyó solo las comunidades sin problemas de seguridad para los estudiantes. Durante el proceso de revisión y depuración de los datos, se pudo haber incurrido en algún tipo de sesgo. Para minimizar esta situación, se incluyeron instrumentos con información completa y adecuada, de acuerdo con los criterios establecidos en el protocolo, lo que permitió registrar una muestra adecuada en tamaño, superando el tamaño muestral estimado para este estudio.

En conclusión, la prevalencia de discapacidad identificada en personas adulto mayor de $51.2 \%$ es alta en comparación con los datos previos de prevalencia nacional de 19.0\% (INE $2002)^{10}$ y global de $43.4 \%$ (OMS 2011). ${ }^{2}$ La capacidad de moverse $(93.0 \%)$ y actividades de la vida diaria (83.0\%), fueron mayormente limitadas contribuyendo a restringir la participación del adulto mayor en la sociedad. Las funciones y estructuras relacionadas al movimiento y sistema cardiovascular se asociaron a discapacidad en el sexo femenino. En hombres, la mayor afectación fue en el sistema respiratorio. La discapacidad se asoció mayormente al antecedente de enfermedad crónica (HTA, artropatía, diabetes mellitus, enfermedad cardiovascular y EPOC) y factores sociodemográficos como violencia, pertenecer al grupo etario $\geq 70$ años, accidentes y caídas en los últimos doce meses y bajo nivel de escolaridad, siendo prevenibles algunas de ellas. Se recomienda profundizar en el análisis de la magnitud de la asociación de los factores identificados, lo cual contribuirá a su mayor comprensión para el diseño de estrategias de promoción y prevención.

Aunque el país cuenta con un amplio marco legal y regulatorio, es necesario fortalecer la formulación y aplicación de políticas públicas a favor del bienestar integral de los adultos mayores mediante la implementación de programas de envejecimiento saludable basado en la participación comunitaria dirigido a la prevención, diagnóstico y manejo de la rehabilitación basada en la comunidad. ${ }^{30-32}$ Debido al impacto que la pandemia por COVID-19 ha provocado en el sistema de salud, la reducción del acceso a los servicios de rehabilitación puede comprometer gravemente la salud y el funcionamiento de grupos más vulnerables como los adultos mayores. ${ }^{33}$ Es importante promover la formación de personal técnico y profesional para la atención del adulto mayor y hacer frente a la discapacidad a través de la rehabilitación basada en la comunidad. ${ }^{34,35}$

\section{CONTRIBUCIONES}

MAM, PMR, MU, LS, SF, CM, SR, LZ concibieron el estudio. MAM, PMR, MU, LS, SF, CM, SR, LZ, JA, MG, EM desarrollaron el diseño metodológico. Todos los autores participaron en el análisis de los resultados. MAM, JA, EM y JS lideraron la escritura del artículo. Todos los autores aprobaron la versión final del artículo y atendieron las recomendaciones editoriales.

\section{AGRADECIMIENTOS}

Se reconoce y agradece la participación de los estudiantes de último año de la Carrera de Medicina periodo 2014-2015, Facultad de Ciencias Médicas UNAH, quienes recolectaron la información del estudio y completaron todas las fases de control de calidad de la información.

\section{DETALLES DE LOS AUTORES}

${ }^{1}$ Melissa A. Mejía meli_mcmejia@yahoo.com

2Paola M. Riverariverapaola83@gmail.com

${ }^{3}$ Melvin Urbina drurbina77@yahoo.com

4Leonardo Sierra faraonramsesii@yahoo.com

${ }^{5}$ Sonia Flores soflorel62@gmail.com

${ }^{4}$ Claudia Martínez claudiaml81@yahoo.es

${ }^{5}$ Saira Rápalo_drasaira@yahoo.com

'Lucia Zúniga_lazi09@hotmail.com

4José E. Sierra capitan1082@hotmail.com

7Jackeline Alger jackelinealger@gmail.com

${ }^{8}$ Mauricio Gonzales gonzalesmauricio721@gmail.com

${ }^{9} E d n a$ Maradiaga edjamar3006@yahoo.com

1-3Residente Postgrado de Medicina de Rehabilitación, Facultad de Ciencias Médicas (FCM), Universidad Nacional Autónoma de Honduras (UNAH), Cohorte 2013-2015. 
1. Organización Mundial de la Salud. Clasificación internacional del funcionamiento de la discapacidad y de la Salud: CIF. [Internet]. Madrid: OPS; Ministerio de Trabajo y Asuntos Sociales. Secretaría General de Asuntos Sociales. Instituto de Migraciones y Servicios Sociales (IMSERSO); 2001. [consultado enero 2021]. Disponible en: https://www.imserso.es/InterPresent2/groups/imserso/documents/binario/435cif.pdf

2. Organización Mundial de la Salud. Informe Mundial sobre la Discapacidad [Internet]. Ginebra: OMS; 2011 [consultado enero 2021]. Disponible en: https://www.who.int/disabilities/world report/2011/es/

3. Menéndez J, Guevara A, Arcia N, León Díaz EM, Marín C, Alonso JC. Enfermedades crónicas y limitación funcional en adultos mayores: estudio comparativo en siete ciudades de América Latina y el Caribe. Rev Panam Salud Pública [Internet]. 2005 [consultado enero 2021];17(5/6):353-61. Disponible en: https://scielosp.org/article/rpsp/2005.v17n5-6/353-361/es/

4. Amate AE, Vásquez AJ, Editores. Discapacidad: lo que todos debemos saber. [Internet]. Washington: OPS; 2006 [consultado enero 2021]. Disponible en: https://iris.paho.org/bitstream/handle/10665.2/719/9275316163. pdf? sequence $=1$ \&isAllowed=y

5. Díaz Calzada M, Delgado A, Naranjo Ferregut JA, Dot Pérez L, Pérez Martín MM. Comportamiento de las discapacidades en los adultos mayores. Consejo Popular Hermanos Barcón. Rev Ciencias Médicas [Internet]. 2012 [consultado enero 2021];16(1):105-117. Disponible en: http://revcmpinar.sld.cu/index.php/publicaciones/article/view/875/html

6. Instituto Nacional de Estadística y Geografía (MX). Perfil sociodemográfico de adultos mayores. [Internet]. México: INEGI; 2014 [consultado enero 2021]. Disponible en: https://n9.cl/dd8sn

7. Stang Alva MF. Las personas con discapacidad en América Latina: del reconocimiento jurídico a la desigualdad real. [Internet]. Santiago: CEPAL; 2011 [consultado enero 2021]. Disponible en: https://repositorio.cepal.org/ bitstream/handle/11362/7135/S1100074 es.pdf

8. Manrique-Espinoza B, Salinas-Rodriguez A, Moreno-Tamayo K, TéllezRojo M. Prevalencia de dependencia funcional y su asociación con caídas en una muestra de adultos mayores pobres en México. Salud Pública Méx [Internet]. 2011 [consultado enero 2021];53(1):26-33. Disponible en: http://www.scielo.org.mx/scielo.php?script=sci arttext\&pid $=$ S0036-36342011000100005

9. Velásquez García GC, Montes Castillo ML, Mazadiego González ME. Asociación del síndrome de temor a caerse, actividades y participación en los adultos mayores. Rev Mex Med Fis Rehab [Internet]. 2013 [consultado enero 2021];25(2):43-48. Disponible en: http://www.medigraphic.com/pdfs/ fisica/mf-2013/mf132b.pdf

10. Instituto Nacional de Estadística (HN). Encuesta permanente de hogares de propósitos múltiples. [Internet]. Tegucigalpa: INE; 2003 [consultado enero 2021]. Disponible en: http://170.238.108.229/index.php/catalog/31

11. Secretaría de Salud (HN).. Encuesta nacional de salud y demografía 2011-2012 [Internet]. Tegucigalpa: INE; 2013. [consultado enero 2021] Disponible en: https://www.ine.gob.hn/publicaciones/endesa/HondurasENDESA-2011-2012.pdf

12. World Health Organization. Measuring health and disability: manual for WHO disability assessment schedule (WHODAS 2.0) [Internet]. Ginebra: WHO; 2010 [consultado enero 2021]. Disponible en: http://www.who.int/ classifications/icf/whodasii/en/\#

13. Vázquez-Barquero JL, Herrera Castanedo S, Vázquez Bourgon E, Gaite Pindado L. Cuestionario para la evaluación de la discapacidad de la Organización Mundial de la Salud (Versión española del World Health Organization Disability Assessment Schedule II): WHO-DAS II. [Internet]. Madrid: Ministerio de Trabajo y Asuntos Sociales; 2006 [consultado enero 2021]. Disponible en: $h$ ttp://sid.usal.es/17646/8-1

14. Huete García A. Autonomía e inclusión de las personas con discapacidad en el ámbito de protección social. Washington: BID; 2019.

15. Instituto Nacional de Estadística e Informática (PE). Característica de la población con discapacidad. [Internet]. Lima: INEI; 2015 [consultado enero 2021]. Disponible en: https://www.inei.gob.pe/media/MenuRecursivo/publicaciones digitales/Est/Lib1209/Libro.pdf
16. Hajjioui A., ABda N., Guenouni R., Nejjari C., Fourtassi M. Prevalence of disability in Morocco: results from a large-scale national survey. J Rehabil Med. 2019;51:508-12

17. De la Fuente-Bacelis TJ, Quevedo-Tejero EC, Jiménez-Sastré A, ZavalaGonzález MA . Funcionalidad para las actividades de la vida diaria en el adulto mayor en zonas rurales. Arch Med Fam [Internet]. 2010 [consultado enero 2021];12(1):1-4. Disponible en: http://www.redalyc.org/ pdf/507/50719083001.pdf

18. Neves-Silva P, Álvarez-Martin E. Estudio descriptivo de características sociodemográficas de la discapacidad en América Latina. Ciênc saúde coletiva [Internet]. 2014 [consultado enero 2021];19(12):4889-4898. Disponible en: http://www.scielo.br/scielo.php?script=sci_arttext\&pid $=$ S1413-81232014001204889

19. Paolinelli C, González M. Epidemiología de la discapacidad en Chile, niños y adultos. Rev Med Clin Condes [Internet]. 2014 [consultado enero 2021];25(2):177-182. Disponible en: https://n9.cl/xfoo

20. Manrique-Espinoza B, Salinas-Rodríguez A, Moreno-Tamayo KM, AcostaCastillo I, Sosa-Ortiz AL, Gutiérrez-Robledo LM, et al. Condiciones de salud y estado funcional de los adultos mayores en México. Salud Pública Méx [Internet]. 2013 [consultado enero 2021];55(suppl 2):S323-S331. Disponible en: http://www.scielo.org.mx/scielo.php?script=sci_arttext\&pid=S0036$36342013000800032 \&$ Ing $=$ es

21. Pinilla-Roncancio M. The reality of disability: multidimensional poverty of people with disability and their families in Latin America. Disabil Health J [Internet]. 2018 [Consultado enero 2021];11(3):398-404. Disponible en: https://doi.org/10.1016/i.dhjo.2017.12.007 Acceso enero 2021

22. HelpAge International. Índice global de envejecimiento, AgeWatch 2015: resumen ejecutivo. [Internet]. Londres: HelpAge International; 2015 [consultado enero 2021]. Disponible en http://www.helpage.org/silo/files/gawi2015-resumen-ejecutivo-.pdf

23. Matus-López M, Cid Pedraza C. Costo de un sistema de atención de adultos mayores dependientes en Chile, 2012-2020. Rev Panam Salud Pública [Internet]. 2014 [consultado enero 2021];36(1):31-36. Disponible en www. scielosp.org/pdf/rpsp/v36n1/05.pdf

24. Nusselder WJ, Wapperom D, Looman CWN, Yokota RTC, van Oyen $\mathrm{H}$, Jagger $\mathrm{C}$, et al. Contribution of chronic condition to disability in men and women in France. Eur J Public Health. 2018; 29(1):99-104.

25. Organización Panamericana de la Salud. Prevención de las enfermedades cardiovasculares: directrices para la evaluación y el manejo del riesgo cardiovascular. [Internet]. Washington: OPS: 2010 [consultado enero 2021]. Disponible en: https://www.paho.org/hq/dmdocuments/2011/Directricespara-evaluacion-y-manejo-del-riego-CV-de-OMS.pdf

26. Bocanegra García LM, Bocanegra García GE, Díaz Camacho PS. Prevalencia y factores biopsicosociales de discapacidad en adultos mayores de los distritos Mesones Muro e Incahuasi, Perú. UCV Scientia [Internet]. 2009 [consultado enero 2021];1(1):84-95. Disponible en: http://repositoriocdpd.net:8080/handle/123456789/284

27. Bogart KR. The role of Disability self-concept in adaptation to congenital or acquired disability. Rehabil Psychol. 2014:59(1):107-15.

28. Mejía MA, Rivera PM, Urbina M, Alger J, Flores S, Maradiaga E, et al. Medicina de rehabilitación: reseña histórica y marco regulatorio en Honduras. Rev Méd Hondur [Internet]. 2014 [consultado enero 2021];82(4):168-174. Disponible en http://www.bvs.hn/RMH/pdf/2014/pdf/Vol82-4-2014-9.pdf

29. Flores $\mathrm{S}$, Jiménez Dilworth AJ, Castellanos Dubón W, Alger J, Zúñiga L, Gonzales M, et al. Prevalencia de discapacidad y sus características en población de 18 a 65 años de edad, Honduras, Centro América, 20132014. Rev Méd Hondur [Internet]. 2015 [consultado enero 2021];83(1-2):717. Disponible en: http://www.bvs.hn/RMH/pdf/2015/pdf/Vol83-1-2-2015-3. pdf

30. Organización Mundial de la Salud. Informe Mundial sobre el envejecimiento y la salud [Internet]. Ginebra: OMS; 2015 [Consultado enero 2021]. Disponible en: https://www.who.int/ageing/publications/world-report-2015/es/

31. Calle Carrasco A, Rolack Jaramillo S, Anguita Mackay V, Campillay Campillay M. Perspectiva ética de la rehabilitación basada en la comunidad 
en el contexto chileno. Rev Bioética y Derecho [Internet]. 2019 [consultado enero 2021];46:185-202. Disponible en: http://scielo.isciii.es/scielo. php?script=sci_arttext\&pid=S1886-58872019000200012\&lng=es\&nrm=i so

32. Organización Mundial de la Salud. Rehabilitación basada en la comunidad (RBC) [Internet]. Ginebra: OMS; 2012 [consultado enero 2021]. Disponible en: https://www.who.int/disabilities/cbr/es/ Acceso enero 2021

33. Organización Panamericana de la Salud. Consideraciones relativas a la rehabilitación durante el brote de COVID-19. Washington: OPS; 2020.

34. Mason C, Weber J, Atasoy S, Sabariego C, Cieza A. Development of indicators for indicators for monitoring Community-Based Rehabilitation. Plos One [Internet]. 2017 [consultado enero 2021];12(6):e0178418. Disponible en: https://pubmed.ncbi.nlm.nih.gov/28575102/

35. Organización Mundial de la Salud. Acción multisectorial para un envejecimiento saludable basado en el ciclo de vida: proyecto de estrategia y plan de acción mundial enero 2021]. Disponible en: https://apps.who.int/ iris/handle/10665/251185
ABSTRACT. Background: The global prevalence of disability in older adults ranges between $29.5 \%$ and $43.4 \%$ in high/low-income countries (WHO 2011). Objective: To determine the prevalence of disability and associated factors in older adults, Honduras, 20142015. Methods: Cross-sectional descriptive study with association analysis. Last year medical students, Universidad Nacional Autónoma of Honduras, actively searched for 50 participants $\geq 60$ years old. Two instruments were applied, one for active search and the other to characterize the participant with/without permanent disability $\geq 1$ year of evolution. Activity limitation and participation restriction $\leq 30$ days were evaluated using the International Classification of Functioning, Disability and Health (ICF) and WHODAS 2.0 questionnaire (WHO). We carried out univariate analysis (frequencies, percentages, prevalences, $\mathrm{Cl} 95 \%$ ) and bivariate analysis (difference in proportions); $p<0.05$ was considered significant). Results: Of a total of 5,126 participants $\geq 60$ years of age, 3,017 $(58.9 \%)$ women, average age 73.6 years (range $60-106)$, the prevalence of disability was $51.2 \%(2,627$ / 5,126); mild degree disability occurred in $1,893(72.1 \%)$. Structures related to movement $(75.5 \%)$ and skeletal function $(72.6 \%)$ were the most affected. The limitation of movement $(93.4 \%)$ and the restriction on investing personal and family money in their health status (86.8\%) were the most frequent. The factors female sex, age $>70$ years old, $<6$ year schooling, chronic illness, trauma, accident, or violence could be associated with disability; all $p<0.01$. Discussion: Disability in older adults was associated with some preventable conditions. It is urgent to implement healthy aging programs in Honduras.

Keywords: Aged; Chronic disease; Disability evaluation; Disabled persons; International Classification of Functioning, Disability and Health. 